\title{
The noncoding explosion
}

\section{The long-held view that the primary role of RNA is to code for proteins has been severely undermined. This Focus explores the remarkable functional diversity of RNA in light of recent breakthroughs in noncoding-RNA biology.}

n 1958, Francis Crick postulated the 'central dogma' to describe the flow of genetic information from DNA to RNA to protein (F.H. Crick, Symp. Soc. Exp. Biol. 12, 138-163, 1958). Experimental evidence then established the mechanistic pathway linking genes to proteins: mRNAs act as transitory templates, tRNAs serve as adaptors between nucleotide and amino acid sequences, and the ribosome functions as the molecular machine that drives protein synthesis. This body of work cemented a canonical view of RNA as primarily a 'coding molecule. Although tRNAs and rRNAs have obvious noncoding functions, their roles are nevertheless intimately tied to translation, thus reinforcing the notion of RNA as template and structural component to aid in protein synthesis.

The finding that RNA itself is capable of enzymatic catalysis in the 1980s jolted the community and eventually led to the 'RNA world' hypothesis, which proposes that self-replicating RNA molecules were precursors to life based on DNA, RNA and proteins. In comparison, the notion of RNA as a regulatory molecule is relatively recent, and the tremendous number, diversity and biological importance of noncoding RNAs (ncRNAs) are only beginning to be fully appreciated. In this issue, we present a special Focus on noncoding RNAs (http://www.nature. $\mathrm{com} / \mathrm{nsmb} /$ focus/noncodingrnas) that explores the functional diversity of ncRNAs, discusses the molecular mechanisms of different RNA interference (RNAi) pathways and highlights the latest breakthroughs in ncRNA biology.

The Focus starts by tying back to the beginning with a Review by Lafontaine ( $\mathrm{p}$ 11) discussing the roles of ncRNAs during the complex process of ribosome biogenesis. That the ribosome is dependent on ncRNA to catalyze critical steps of mRNA decoding and amino acid polymerization is, of course, old news. rRNA constitutes the functional core of the ribosome, whereas another group of ncRNAs, small nucleolar RNAs (snoRNAs), controls rRNA abundance and quality. Interestingly, recent evidence has indicated that snoRNAs contribute to unexpected ribosome heterogeneity, with likely consequences for ribosome function.

It was, however, the discovery of RNAi in the 1990s that placed ncRNAs firmly in the limelight. In this process, small ncRNAs, such as microRNAs (miRNAs), small interfering RNAs (siRNAs) and Piwiinteracting RNAs (piRNAs), serve as guides to direct molecular machinery to target mRNAs in order to silence gene expression. Silencing occurs through direct endonucleolytic cleavage, destabilization of the mRNA target or transcriptional or translational repression. There has been great progress in elucidating the molecular mechanisms that govern different RNAi pathways, and Ipsaro and Joshua-Tor (p 20) review our current knowledge on this topic, exploring the structure and modes of RNAi biogenesis and effector machinery. The identification of small ncRNAs capable of specifically silencing virtually any gene encoded in the genome has revolutionized the ability to probe biological systems.
A deeper mechanistic understanding of the modes of target recognition and silencing should increase applications even further.

Underpinning the notion of ncRNAs as regulatory molecules was the revelation that the vast majority of the cellular transcriptome is actually dedicated to diverse types of ncRNAs—a discovery made possible by postgenomic deep-sequencing technologies. Of these, long noncoding RNAs (lncRNAs) are emerging as a class of key regulators that can affect gene expression, either through direct interference with RNA polymerases or by targeting chromatin-modifying enzymes. They have also been reported to act as molecular decoys, to scaffold protein-protein interactions and to affect nuclear organization.

Thousands of lncRNAs have been discovered to date, but their functional characterization has remained a challenge. This is partly because of a shortage of experimental techniques to explore their functions. In their Perspective, Spitale, Chang and Chu (p 29) highlight recent technological advances that will aid in the functional characterization of lncRNAs and discuss their advantages and caveats. The sheer number and the increasing pace of the discovery of new lncRNAs also present a challenge in terms of lncRNA definition and annotation. This issue is addressed in a Commentary by Rinn and Mattick ( $\mathrm{p} 5$ ), who propose considerations and best practices for identifying and annotating lncRNAs. These guidelines should assist the growing research community embarking on the mechanistic investigation of lncRNAs.

Given their immense regulatory potential, it is not surprising that ncRNAs have been shown to affect many physiological, developmental and disease processes. In their Commentary, Li and Izpisua Belmonte ( $\mathrm{p} 2$ ) discuss the relevance of ncRNAs in cell-fate determination and reprogramming. Similarly to protein factors, ncRNAs are able to remodel transcriptional circuits and reshape epigenetic landscapes that determine cellular identity. These features can be exploited to drive cellfate switching and, ultimately, tissue regeneration.

The unique structural and biochemical properties of RNA also make it an ideal substrate for bioengineering. The predictable base-pairing of RNA allows for the construction of a wide range of structures that can act as scaffolds for the localization of enzymes and other molecules. Just like their natural counterparts, synthetic RNAs can affect gene expression through a number of distinct mechanisms and can thus be used as regulators of synthetic gene circuits. These exciting applications are highlighted in a Commentary by Myhrvold and Silver (p 8).

Considering the boom in ncRNA research and the many open questions faced by the community of RNA researchers, it is clear that exciting times lie ahead. We are eager to see advances emerge and develop within the pages of Nature Structural \& Molecular Biology. Last, but not least, we thank all contributors to this Focus. We hope that you will enjoy the read! 